\title{
A GENERALIZATION OF A THEOREM BY D. K. FADDEEV
}

\section{KONRAD BEHNEN}

ABSTRACT. In this paper we give a simple proof of the statement $\lim _{n \rightarrow \infty} \int K_{n}(x, y) f(y) d \mu(y)=f(x)$ for $\mu$-almost all $x$ under weaker and more general assumptions than those of the usual $\mathrm{Faddeev}$ theorems.

1. Introduction and summary. In nonparametric statistics the problem arises of generating scores $b_{n i}$ or $b_{n i j}$ in such a way that

or

$$
b_{n}=\sum_{i=1}^{n} b_{n i} I_{[(i-1) / n, i / n)}
$$

$$
b_{n}=\sum_{i, j} b_{n i j} I_{[(i-1) / n, i / n) \times[(j-1) / n, j / n)}
$$

converge (in quadratic mean) to some (square) integrable function $b$ defined on $(0,1)$ or $(0,1) \times(0,1)$, respectively. In the one-dimensional case Hajek and Šidák [4] used a theorem by D. K. Faddeev (cf. Natanson [5, Theorem X.2.3] or Dunford and Schwartz [2, Theorem III.12.11]) to solve this problem for the special scores $b_{n i}=\int_{0}^{1} b(x) f_{n i}(x) d x$, where $f_{n i}$ denotes the $\beta$-density $n\left(\begin{array}{c}n-1 \\ i-1\end{array}\right) x^{i-1}(1-x)^{n-i}$ on $(0,1)$. In the two-dimensional case (and similarly for higher dimensions) it was shown in [1] that the same result holds for the scores

$$
b_{n i j}=\int_{0}^{1} \int_{0}^{l} b(x, y) f_{n i}(x) f_{n j}(y) d x d y .
$$

However, the proof is based on probabilistic arguments and rests heavily on the special form of the scores considered. Therefore it may not work for more general scores. Thus, one is interested in a generalization of Faddeev's theorem to higher dimensions.

Another application may be the construction of density estimators in a more general setting than $k$-dimensional Euclidean space with Lebesguemeasure.

First, we shall give a much simpler proof (even under weaker conditions)

Received by the editors November 1, 1972 and, in revised form, September 18, 1973. AMS (MOS) subject classifications (1970). Primary $28 \mathrm{~A} 15$. 
of the "classical" theorem as stated in Dunford and Schwartz [2] to show the simplicity of the argument. Second, we shall establish the theorem for general metric spaces and then we shall give some examples which include the above-mentioned example.

2. Results. We cite the special theorem in its given form, but one should note that the condition (b) of the theorem is not needed in our proof.

Theorem 1 (Dunford and Schwartz, Vol. I, Theorem III. 12.11). Let $\left\{K_{n}\right\}$, $\left\{R_{n}\right\}$ be sequences of scalar functions defined on the plane with $R_{n}$ real valued and $\left|K_{n}(s, t)\right| \leq R_{n}(s, t)$. Suppose that for each fixed $s$ we have

(a) $R_{n}(s, t)$ is decreasing as a function of $t$ when $t \geq s$, is increasing when $t \leq s$ and is continuous on the right;

(b) $\lim _{|t| \rightarrow \infty} R_{n}(s, t)=0$;

(c) there is a constant $M(s)$ such that $\int_{s-1}^{s+1} R_{n}(s, t) d t \leq M(s) \forall n$;

(d) $\lim _{n \rightarrow \infty} \int_{s-1}^{s+1} K_{n}(s, t) d t=1$;

(e) $\lim _{n \rightarrow \infty} R_{n}(s, t)=0$ for each pair $(s, t)$ with $s \neq t$.

Then if $f$ is a Lebesgue integrable function defined for $-\infty<s<\infty$ with values in a Banach space. $\mathfrak{X}$,

$$
\lim _{n \rightarrow \infty} \int_{-\infty}^{+\infty} K_{n}(s, t) f(t) d t=f(s)
$$

for every $s$ in the Lebesgue set of $f$.

Remark. The Lebesgue set of $f$ is defined as the set of all points $s$ at which

$$
\lim _{\lambda_{1}(C) \rightarrow 0} \frac{1}{\lambda_{1}(C)} \int_{C}|f(t)-f(s)| d t=0,
$$

where $C$ is a closed interval containing $s$. It is well known that the Lebesgue set of $f$ contains $\lambda_{1}$-almost all points.

Proof. The integral $\int_{-\infty}^{+\infty} K_{n}(s, t) f(t) d t$ exists for each $n$, since the monotonicity of $R_{n}(s, \cdot)$ on $(-\infty, s]$ and on $[s, \infty)$ implies that $R_{n}(s, \cdot)$ and therefore $\left|K_{n}(s, \cdot)\right|$ is bounded for each $n$. On the other hand, for each $s$ and each $\epsilon>0$, we get from (a) and (e)

$0 \leq\left|\int_{(s-\epsilon, s+\epsilon) c} K_{n}(s, t) f(t) d t\right| \leq \sup _{|s-t| \geq \epsilon} R_{n}(s, t) \int|f(t)| d t \rightarrow 0 \quad(n \rightarrow \infty)$, 
$0 \leq\left|\int_{[s-1, s+1] \cap(s-\epsilon, s+\epsilon)^{c}} K_{n}(s, t) d t\right| \leq 2 \sup _{|s-t| \geq \epsilon} R_{n}(s, t) \rightarrow 0 \quad(n \rightarrow \infty)$.

Thus, for each $s$, we may choose a sequence $\left\{\epsilon_{n}(s)\right\}$ with $0<\epsilon_{n}(s) \leq 1$, $\epsilon_{n}(s) \rightarrow 0(n \rightarrow \infty)$, such that the two relations stated above remain true, if we substitute $\epsilon$ by $\epsilon_{n}(s)$. Keeping this in mind, we derive from (d):

$\int_{-\infty}^{+\infty} K_{n}(s, t) f(t) d t$

$$
\begin{aligned}
& =o(1)+\int_{s-\dot{\epsilon}_{n}(s)}^{s+\epsilon_{n}(s)+} K_{n}(s, t)(f(t)-f(s)) d t+\int_{s-\epsilon_{n}(s)}^{s+\epsilon_{n}(s)} K_{n}(s, t) d t f(s) \\
& =f(s)+o(1)+\int_{s-\epsilon_{n}(s)}^{s+\epsilon_{n}(s)} K_{n}(s, t)(f(t)-f(s)) d t .
\end{aligned}
$$

Thus it suffices to prove $\int_{s-\epsilon_{n}(s)}^{s+\epsilon_{n}(s)} R_{n}(s, t)|f(t)-f(s)| d t \rightarrow 0(n \rightarrow \infty)$ for each $s$ in the Lebesgue set of $f$. Now take such an $s$. Then by (a) and Fubini

$$
\begin{aligned}
0 & \leq \int_{s-\epsilon_{n}(s)}^{s+\epsilon_{n}(s)} R_{n}(s, t)|f(t)-f(s)| d t \\
& =\int_{s-\epsilon_{n}(s)}^{s+\epsilon_{n}(s)}\left(\int_{0}^{R_{n}(s, s)} I_{\left[0, R_{n}(s, t)\right]}(r) d r\right)|f(t)-f(s)| d t \\
& =\int_{0}^{R}{ }^{(s, s)}\left(\int_{s-\epsilon_{n}(s)}^{s+\epsilon_{n}(s)} I_{[r, \infty)}\left(R_{n}(s, t)\right)|f(t)-f(s)| d t\right) d r \\
& \leq o(1) \int_{0}^{R_{n}(s, s)} \lambda_{1}\left(\left[s-\epsilon_{n}(s), s+\epsilon_{n}(s)\right] \cap\left\{t: R_{n}(s, t) \geq r\right\}\right) d r
\end{aligned}
$$

since for each $r$ the set $\left[s-\epsilon_{n}(s), s+\epsilon_{n}(s)\right] \cap\left\{t: R_{n}(s, t) \geq r\right\}$ is empty for an interval $J_{s n}(r)$ with $s \in J_{s n}(r)$ and $\lambda_{1}\left(J_{s n}(r)\right) \leq 2 \epsilon_{n}(s)(\rightarrow 0(n \rightarrow \infty))$ and since $s$ is in the Lebesgue set of $f$. Finally we get again by Fubini and (c):

$$
\begin{aligned}
0 & \leq \int_{0}^{R_{n}(s, s)} \lambda_{1}\left(\left[s-\epsilon_{n}(s), s+\epsilon_{n}(s)\right] \cap\left\{R_{n}(s, t) \geq r\right\}\right) d r \\
& =\int_{0}^{R_{n}(s, s)}\left(\int_{s-\epsilon_{n}(s)}^{s+\epsilon_{n}(s)} I_{[r, \infty)}\left(R_{n}(s, t)\right) d t\right) d r \\
& =\int_{s-\epsilon_{n}(s)}^{s+\epsilon_{n}(s)}\left(\int_{0}^{R_{n}(s, s)} I_{\left[0, R_{n}(s, t)\right]}(r) d r\right) d t \\
& \leq \int_{s-1}^{s+1} R_{n}(s, t) d t \leq M(s) \quad \forall n .
\end{aligned}
$$

Before stating the general theorem we list the assumptions which are made throughout this section and give some definitions used in the theorem:

Let $(X, \hat{U}, \mu)$ be a positive measure space, where $X$ is a metric space with metric $\rho$, $\mathcal{U}$ is the Borel $\sigma$-field on $X$, and $\mu$ is a (positive) measure on 
If such that every bounded measurable set has finite measure. Let $Y$ be a Banach space with its Borel $\sigma$-field and let $\left\{K_{n}\right\},\left\{R_{n}\right\}$ be sequences of scalar-valued functions defined on $X \times X$ with $R_{n}$ real valued, $K_{n}(x, \cdot)$ and $R_{n}(x, \cdot)$ Borel measurable for each $x \in X$ and $n \in \mathbf{N}$, and

$$
\left|K_{n}(x, y)\right| \leq R_{n}(x, y) \quad \forall x, y \in X, \quad \forall n \in \mathbf{N} .
$$

Definition. (Cf. Federer $[3,2.8 .16]$.) (a) Every subset $B$ of $\{(x, S)$ : $x \in S \subset X\}$ is called a covering relation.

(b) If $B$ is a covering relation and $Z \subset X$, we denote by $B(Z)$ the set $\{S:(x, S) \in \mathfrak{B}$ for some $x \in Z\}$.

(c) A covering relation $\mathfrak{B}$ is called fine at $x$ if $\inf \{\operatorname{diam}(S):(x, S) \in \mathbb{B}\}$ $=0$, where $\operatorname{diam}(S)$ denotes $\sup \{\rho(y, z): y, z \in S\}$.

(d) $B$ is called a $\mu$-Vitali relation if $B$ is a covering relation such that $B(X)$ is a family of Borel sets, $B$ is fine at each point of $X$, and the following condition holds: if $\mathbb{S} \subset \mathbb{B}, Z \subset X$ and $\mathbb{S}$ is fine at each point of $Z$, then $\mathcal{S}(Z)$ has a countable disjointed. subfamily covering $\mu$-almost all of $Z$.

Theorem 2. For each fixed $x \in X$ assume the existence of a sequence $\left\{\epsilon_{n}(x)\right\}$ with $0<\epsilon_{n}(x) \rightarrow 0(n \rightarrow \infty)$ and the existence of $M(x)<\infty$ such that

$$
\begin{aligned}
& \int_{B\left(x, \epsilon_{n}(x)\right)} K_{n}(x, y) d \mu(y) \rightarrow 1 \quad(n \rightarrow \infty), \\
& \int_{B\left(x, \epsilon_{n}(x)\right)} R_{n}(x, y) d \mu(y) \leq M(x) \quad \forall n \in \mathbf{N},
\end{aligned}
$$

where $B\left(x, \epsilon_{n}(x)\right)$ is the closed ball with centre $x$ and radius $\epsilon_{n}(x)$. For each $n \in \mathbf{N}, r \geq 0, x \in X$ put

and suppose

$$
A(n, r, x):=\left\{y: R_{n}(x, y) \geq r\right\} \cap B\left(x, \epsilon_{n}(x)\right)
$$

(3) $\mathscr{B}:=\left\{(x, A(n, r, x)): 0 \leq r<\widetilde{R}_{n}(x):=\sup _{y \in B\left(x, \epsilon_{n}(x)\right)} R_{n}(x, y), x \in X, n \in \mathrm{N}\right\}$ is a $\mu$-Vitali relation.

Finally, let $f \in L_{1}(X, \hat{U}, \mu, Y)$ such that

$$
\int_{X-B\left(x, \epsilon_{n}(x)\right)} K_{n}(x, y) f(y) d \mu(y) \rightarrow 0(n \rightarrow \infty) \quad \forall x \in X .
$$

Then $\int K_{n}(x, y) f(y) d \mu(y) \rightarrow f(x)(n \rightarrow \infty)$ for $\mu$-almost all $x \in X$, in particular for all $x$ in the ( $\mu, B)$-Lebesgue set of $f$ (cf. Federer [3, 2.9.9]).

Proof. (3) implies $x \in A(n, r, x) \forall r<\tilde{R}_{n}(x) \forall n$. This means $\tilde{R}_{n}(x)=$ $R_{n}(x, x)<\infty$, i.e. $R_{n}(x, \cdot)$ is bounded on $B\left(x, \epsilon_{n}(x)\right)$. Thus (1) and (4) imply the existence of $\int K_{n}(x, y) f(y) d \mu(y)$. From (2) and (4) we get 


$$
\begin{aligned}
\int K_{n}(x, y) f(y) d \mu(y)= & \int_{B\left(x, \epsilon_{n}(x)\right)} K_{n}(x, y) d \mu(y) f(x) \\
& +\int_{B\left(x, \epsilon_{n}(x)\right)} K_{n}(x, y)(f(y)-f(x)) d \mu(y) \\
& +\int_{X-B\left(x, \epsilon_{n}(x)\right)} K_{n}(x, y) f(y) d \mu(y) \\
= & f(x)+o(1)+\int_{B\left(x, \epsilon_{n}(x)\right)} K_{n}(x, y)(f(y)-f(x)) d \mu(y) .
\end{aligned}
$$

Because of (1) and because of Federer [3, Corollary 2.9.9] it suffices to prove:

$$
\int_{B\left(x, \epsilon_{n}(x)\right)} R_{n}(x, y)|f(y)-f(x)| d \mu(y) \rightarrow 0(n \rightarrow \infty)
$$

for each $x$ in the $(\mu, \mathbb{B})$-Lebesgue set of $f$.

For proving (5) take such an $x$. Then we get from $\sup _{r} \operatorname{diam}(A(n, r, x))$ $\leq 2 \epsilon_{n}(x) \rightarrow 0(n \rightarrow \infty)$, and (3)

$$
\begin{aligned}
& \sup \left\{\int_{A(n, r, x)} \frac{|f(y)-f(x)| d \mu(y)}{\mu(A(n, r, x))}:\right. \\
& \left.\mu(A(n, r, x))>0, r<R_{n}(x, x)\right\}=o(1) .
\end{aligned}
$$

This, together with Fubini and the second in equality in (2), implies the following chain of inequalities which proves (5).

$$
\begin{aligned}
& \int_{B\left(x, \epsilon_{n}(x)\right)} R_{n}(x, y)|f(y)-f(x)| d \mu(y) \\
& =\int_{B\left(x, \epsilon_{n}(x)\right)}\left(\int_{0}^{R}{ }^{(x, x)} I_{\left[0, R_{n}(x, y)\right]}(r) d r\right)|f(y)-f(x)| d \mu(y) \\
& =\int_{0}^{R_{n}(x, x)}\left(\int_{A(n, r, x)}|f(y)-f(x)| d \mu(y)\right) d r \leq o(1) \int_{0}^{R_{n}(x, x)} \mu(A(n, r, x)) d r \\
& =d(1) \int_{0}^{R_{n}(x, x)}\left(\int_{B\left(x, \epsilon_{n}(x)\right)} I_{\left[0, R_{n}(x, y)\right]}(r) d \mu(y)\right) d r \\
& =o(1) \int_{B\left(x, \epsilon_{n}(x)\right)}\left(\int_{0}^{R_{n}(x, y)} d r\right) d \mu(y) \\
& =o(1) \int_{B\left(x, \epsilon_{n}(x)\right)} R_{n}(x, y) d \mu(y) \leq o(1) M(x) \rightarrow 0(n \rightarrow \infty) .
\end{aligned}
$$

Condition (3) is somewhat difficult to handle (cf. Federer $[3,2.8 .17-$ 2.8. 20]). Therefore we will give a corollary easy for applications.

Corollary. For each fixed $x \in X$ let $R_{n}(x, \cdot)$ be upper semicontinuous and assume the existence of an $\epsilon(x)>0$ and an $M(x)<\infty$ such that 


$$
\begin{gathered}
\int_{B(x, \epsilon(x))} K_{n}(x, y) d \mu(y) \rightarrow 1(n \rightarrow \infty), \\
\int_{B(x, \epsilon(x))} R_{n}(x, y) d \mu(y) \leq M(x) \quad \forall n \in \mathbf{N}, \\
R_{n}\left(x, y_{1}\right) \geq R_{n}\left(x, y_{2}\right) \quad \text { if } \rho\left(x, y_{1}\right) \leq \rho\left(x, y_{2}\right), \\
R_{n}(x, y) \rightarrow 0(n \rightarrow \infty) \quad \text { if } y \neq x .
\end{gathered}
$$

Finally assume the existence of $a \beta>3$ such that for $\mu$-almost all $x \in X$ there exists an $\alpha(x)<\infty$ with

$$
\mu(B(x, \beta r)) \leq \alpha(x) \mu(B(x, r)) \quad \forall r \in(0, \epsilon(x)) .
$$

Then for each $f \in L_{1}(X, \mathcal{U}, \mu, Y)$ we have $\int K_{n}(x, y) f(y) d \mu(y) \rightarrow f(x)(n \rightarrow$ $\infty)$ for $\mu$-almost all $x \in X$.

Proof. Because of (7) and (8) we get, for each $x \in X$, the existence of a sequence $\left\{\epsilon_{n}(x)\right\}$ with $0<\epsilon_{n}(x)<\epsilon(x)$ and $\epsilon_{n}(x) \rightarrow 0(n \rightarrow \infty)$ such that

$$
\sup _{y \in X-B\left(x, \epsilon_{n}(x)\right)} R_{n}(x, y) \rightarrow 0(n \rightarrow \infty) .
$$

This, together with (1) and (6), implies (2) and (4) of Theorem 2. Finally, we establish (3) of Theorem 2 in the following way: Because of (7) and the upper semicontinuity of $R_{n}(x, \cdot)$ we have, for each $n \in \mathrm{N}, x \in X, 0 \leq r<$ $R_{n}(x, x)$, that $A(n, r, x)$ is a closed ball with centre $x$ and radius less than or equal to $\epsilon_{n}(x)$. Therefore (9) and Federer [3, Theorem 2.8.17] (with $\delta=$ diam) imply

$$
\mathscr{B}:=\left\{(x, A(n, r, x)): 0 \leq r<R_{n}(x, x), x \in \mathbf{N}\right\}
$$

to be a $\mu$-Vitali relation.

Remark. Because of $\lambda_{k}(B(x, \beta r))=\beta^{k} \lambda_{k}(B(x, r)) \forall \beta, r>0$, for the Lebesgue-measure $\lambda_{k}$ on the $k$-dimensional Euclidean space $\mathbf{R}_{k}$, even the corollary is an obvious generalization of Theorem 1.

3. Examples.

3.1. Let $(X, 21, \mu)=\left(\mathbf{R}_{k}, \mathfrak{B}_{k}, \lambda_{k}\right)$ be the $k$-dimensional Euclidean space with its Borel $\sigma$-field and the Lebesgue-measure. For each $x \in \mathbf{R}_{k}$ let $K_{n}(x, \cdot): \mathbf{R}_{k} \rightarrow[0, \infty)$ be the (continuous) Lebesgue-density of the distribution of $k$ independent normally distributed random variables with mean vector $x$ and variances $\sigma_{n 1}^{2}, \cdots, \sigma_{n k}^{2}$ such that there exists an $\epsilon \epsilon(0,1]$ with

$$
\epsilon \leq \min \left\{\sigma_{n 1}^{2}, \cdots, \sigma_{n k}^{2}\right\} / \max \left\{\sigma_{n 1}^{2}, \cdots, \sigma_{n k}^{2}\right\} \leq 1 / \epsilon
$$


for all $n \in \mathbf{N}$ and $\Sigma_{i=1}^{k} \sigma_{n i}^{2} \rightarrow 0(n \rightarrow \infty)$. Then for each $f \in L_{1}\left(\mathbf{R}_{k}, B_{k}, \lambda_{k}, Y\right)$ we have $\int K_{n}(x, y) f(y) d \lambda_{k}(y) \rightarrow f(x)(n \rightarrow \infty)$ for $\lambda_{k}$-almost all $x \in \mathbf{R}_{k}$ since $\lambda_{k}, K_{n}$, and

$$
\begin{aligned}
R_{n}(x, y):= & (1 / \epsilon)^{k / 2}\left(2 \pi \max \left\{\sigma_{n 1}^{2}, \cdots, \sigma_{n k}^{2}\right\}\right)-k / 2 \\
& \cdot \exp \left(-\left(1 / 2 \max \left\{\sigma_{n 1}^{2}, \cdots, \sigma_{n k}^{2}\right\}\right) \sum_{i=1}^{k}\left(y_{i}-x_{i}\right)^{2}\right)
\end{aligned}
$$

fulfill the conditions of the corollary.

3.2. Let $(X$, OU, $\mu)=\left(\mathbf{R}_{2}, B_{2}, \lambda_{2}\right)$ and for each $x \in \mathbf{R}_{2}$ let $K_{n}(x, \cdot)$ be the (continuous) Lebesgue-density of two normally distributed random variables with mean vector $x$, variances $\sigma_{n 1}^{2}, \sigma_{n 2}^{2}$, and covariance $\rho_{n}$ such that there exists an $\epsilon \epsilon(0,1]$ with $\epsilon \leq \sigma_{n 1}^{2} / \sigma_{n 2}^{2} \leq 1 / \epsilon$ and $\rho_{n}^{2} \leq 1-\epsilon$ for all $n \epsilon$ $\mathbf{N}$ and $\sigma_{n 1}^{2}+\sigma_{n 2}^{2} \rightarrow 0(n \rightarrow \infty)$. Then for each $f \in L_{1}\left(\mathbf{R}_{2}, B_{2}, \lambda_{2}, Y\right)$ we have $\int K_{n}(x, y) f(y) d \lambda_{2}(y) \rightarrow f(x)(n \rightarrow \infty)$ for $\lambda_{2}$-almost all $x \in \mathbf{R}_{2}$ since $\lambda_{2}, K_{n}$, and

$$
\begin{aligned}
R_{n}(x, y):= & \left(2 / \epsilon^{3 / 2}\right)\left(4 \pi \max \left\{\sigma_{1 n}^{2}, \sigma_{2 n}^{2}\right\}\right)^{-1} \\
& \cdot \exp \left(-\left(1 / 4 \max \left\{\sigma_{1 n}^{2}, \sigma_{2 n}^{2}\right\}\right)\left(\left(y_{1}-x_{1}\right)^{2}+\left(y_{2}-x_{2}\right)^{2}\right)\right)
\end{aligned}
$$

fulfill the conditions of the corollary.

3.3. Let $(X, \hat{U}, \mu)$ be the $k$-dimensional unit cube with its Borel $\sigma$ field and the Lebesgue measure $\lambda_{k}$ on it. For each $x=\left(x_{1}, \cdots, x_{k}\right) \in$ $(0,1)^{(k)}$ let

a product of $\beta$-densities

$$
K_{n}(x, y)=\prod_{i=1}^{k} f_{n\left[n x_{i}+1\right]}\left(y_{i}\right)
$$

$$
f_{n i}(s)=n\left(\begin{array}{c}
n-1 \\
i-1
\end{array}\right) s^{i-1}(1-s)^{n-i}, \quad i \in\{1, \cdots, n\},
$$

defined on $(0,1)$. ( $[t]$ denotes as usual the greatest integer less than or equal to $t$.) With the notation

$$
\begin{aligned}
g_{n i}(s)= & f_{n i}(s)\left(1-I_{[(i-1) / n, i / n]}(s)\right) \\
& +n\left(\begin{array}{c}
n-1 \\
i-1
\end{array}\right)\left(\frac{i-1}{n-1}\right)^{i-1}\left(\frac{n-i}{n-1}\right)^{n-i} I_{[(i-1) / n, i / n]}(s)
\end{aligned}
$$

we get (cf. Hájek and Šidák [4, pp. 158-159]). that, for all $i=1, \ldots, n$ and all $n \in \mathbf{N}: f_{n i} \leq g_{n i}, \int_{0}^{1} g_{n i}(t) d t \leq 2, g_{n}[n s+1]^{(\cdot)}$ is increasing on $(0, s]$ and decreasing on $[s, 1) \forall s \in(0,1)$. Furthermore, we have by Stirling's formula 


$$
\sup _{t \in(0,1)} g_{n[n s+1]}(t)=g_{n[n s+1]}([n s] /(n-1)) \leq n^{1 / 2} K(s)<\infty
$$

and

$\forall s \neq t \exists R(s, t)<\infty, p(s, t) \in(0,1): g_{n[n s+1]}(t) \leq n^{1 / 2}(p(s, t))^{n} R(s, t) \quad \forall n \in \mathbf{N}$.

Therefore we have $0 \leq K_{n} \leq R_{n}$ and $R_{n}(x, y) \rightarrow 0(n \rightarrow \infty)$ if $x \neq y$ by putting

$$
R_{n}(x, y):=\prod_{i=1}^{k} g_{n\left[n x_{i}+1\right]}\left(y_{i}\right) \quad \forall x, y \in(0,1)^{(k)}, \quad \forall n \in \mathbf{N} .
$$

Thus, with these $K_{n}$ and $R_{n}$, conditions (1), (2), and (4) of Theorem 2 are true for each $f \in L_{1}(X, \mathcal{U}, \mu, Y)$. For proving condition (3) of Theorem 2 we observe that $x \in A(n, r, x)$ if $0 \leq r<\widetilde{R}_{n}(x), A(n, r, x)$ is a bounded closed set, and $\operatorname{diam}(A(n, r, x)) \rightarrow 0(n \rightarrow \infty)$. Therefore we obtain (3) from Federer [3, Theorem 2.8.17] since for each $x \in X$ there exists an $\alpha(x)<\infty$ such that each $A(n, r, x)$ is contained in a cube $Q(n, r, x)$ with

$$
\lambda_{k}(Q(n, r, x)) \leq \alpha(x) \lambda_{k}(A(n, r, x)) \quad \forall 0 \leq r<\tilde{R}_{n}(x), \quad \forall n \in \mathbf{N} .
$$

The last statement is true because, with properly chosen $\epsilon_{n}(x)$,

$$
A(n, r, x) \supset \underset{i=1}{\times}\left[\left[n x_{i}\right] / n,\left[n x_{i}+1\right] / n\right] \quad \forall 0 \leq r<\widetilde{R}_{n}(x), \quad \forall n \in \mathbf{N},
$$

and (by Stirling's formula)

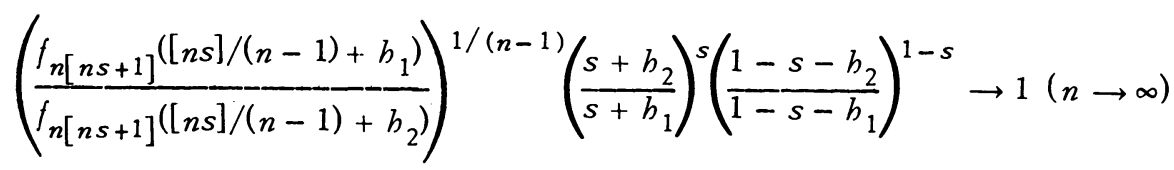

uniformly in $h_{1}, h_{2} \in[-s / 2,(1-s) / 2]$.

\section{REFERENCES}

1. K. Behnen, A characterisation of certain rank-order tests with bounds for the asymptotic relative efficiency, Ann. Math. Statist. 43 (1972), 1839-1851.

2. N. Dunford and J. T. Schwartz, Linear operators. I: General theory, Pure and Appl. Math., vol. 7, Interscience, New York, 1958. MR 22 \#302.

3. H. Federer, Geometric measure theory, Die Grundlehren der math. Wissenschaften, Band 153, Springer-Verlag, New York, 1969. MR 41 \#1976.

4. J. Hájek and Z. Šidák, Theory of rank tests, Academic Press, New York; Academia, Prague, 1967. MR 37 \#425.

5. I. P. Natanson, Theorie der Funktionen einer reellen Veränderlichen, 3rd ed., Akademie-Verlag, Berlin, 1969.

INSTITUT FÜR MATHEMATISCHE STOCHASTIK DER UNIVERSITÄT FREIBURG, 78 FREIBURG, HERMANN-HERDER-STRASSE 10, WEST GERMANY 